\title{
Electron Collision Frequency in the Ionospheric $D$ Region
}

\author{
Robert F. Benson ${ }^{1}$ \\ Contribution From the Geophysical Institute of the University of Alaska, College, Alaska
}

(Received November 18, 1963; revised January 3, 1964)

\begin{abstract}
A brief review of the ionospheric $D$ region electron collision frequency information that is available from rocket observations and laboratory investigations indicates that the equation $v_{\mathrm{m}}=8.40 \times 10^{7} \mathrm{p}(\mathrm{mm} \mathrm{Hg})$ is accurate within about \pm 10 percent in the portion of the $D$ region above $40 \mathrm{~km}$. The results of the cross-modulation experiment at College, Alaska, are in agreement with this equation.
\end{abstract}

\section{Introduction}

The electron collision frequency $\nu$ in the ionospheric $D$ region is an important parameter in the theory of radio wave absorption and in the theory of ionospheric cross modulation, which was discussed in the preceding paper [Benson, 1964]. The purpose of the present paper is to briefly review the pertinent information concerning $\nu$ that is available from rocket observations and laboratory investigations and compare the results with the determinations of $\nu$ from the ionospheric cross modulation observations at College, Alaska.

\section{Laboratory Investigations and Rocket Observations}

To obtain reliable electron collision frequency profiles in the ionosphere from rocket experiments, one must use the generalized magneto-ionic theory, rather than the Appleton-Hartree theory, in the data analysis. The electron collision frequency profiles in the ionosphere, as determined from rocket data, then agree with recent laboratory measurements of the electron collision frequencies in nitrogen [Phelps and Pack, 1959; Kane, 1960].

Phelps [1960] discusses the results of laboratory measurements of the electron collision frequency in nitrogen and oxygen; he also discusses the effect of electron collisions with ions and water vapor molecules. The results of three separate experiments in nitrogen are in very good agreement. Of particular importance to the present discussion is the work of Phelps and Pack [1959] and Pack and Phelps [1961], since their measurements extend down to the electron energy range appropriate to the ionospheric $D$ region. The latter paper gives the best results for $\mathrm{N}_{2}$, and is thus more suitable for ionospheric applications [Phelps, private communication, 1963]. The momentum transfer cross section for electrons in $N_{2}$ is given as

$$
\sigma=u^{1 / 2}\left[5.31 \times 10^{14}-\frac{3.15 \times 10^{11}}{u}\right]^{-1} \mathrm{~cm}^{2}
$$

${ }_{1}^{1}$ Present address: NASA - Goddard Space Flight Center, Greenbelt, Md. where $u=k \theta$ is the thermal energy of an electron associated with the most probable electron velocity $v_{m}$ ( $k$ is Boltzmann's constant in $\mathrm{eV} /{ }^{\circ} \mathrm{K}$ and $\theta$ is the electron temperature in ${ }^{\circ} \mathrm{K}$ ).

For the range of electron energies encountered up to at least $70 \mathrm{~km}$ in high latitude regions $(u \geq 0.017$ $\mathrm{eV}$, i.e., $\theta \geq 200^{\circ} \mathrm{K}$ [Stroud, Nordberg, and Brandeen, 1960]) the following one term approximation for (1) can be used with an error of less than 4 percent:

$$
\sigma=\frac{u^{1 / 2}}{5.31 \times 10^{14}}=1.88 \times 10^{-15} u^{1 / 2} \mathrm{~cm}^{2} .
$$

The electron collision frequency in nitrogen is then given by

$$
\begin{aligned}
\nu_{m} & =n \sigma v_{m}=n \sigma\left(\frac{2 k \theta}{m}\right)^{1 / 2} \\
& =5.93 \times 10^{7} n \sigma u^{1 / 2} \\
& =1.11 \times 10^{-7} n\left(N_{2}\right) u \mathrm{sec}^{-1}
\end{aligned}
$$

where $n\left(N_{2}\right)$ is the nitrogen density in molecules $/ \mathrm{cm}^{3}$. (Note: there is an error in footnote 2a of Phelps [1960] which intended to correct the 1960 results so as to agree with the 1961 results. This footnote should read "The derived electron collision frequencies are 10 to 12 percent smaller than those given in this paper." [Phelps, private communication, 1963].) In (3), $\nu_{m}$ refers to the electron collision frequency for momentum transfer; in (2) of the preceding paper [Benson, 1964], it refers to the electron collision frequency for monoenergetic electrons of an energy equal to $k \theta$; i.e., related to the most probable value of the electron speed. No ambiguity is introduced if $\nu_{m}$ represents both of the above quantities, i.e., the electron collision frequency for momentum transfer for monoenergetic electrons of an energy $k \theta$; this terminology is equivalent to that of Phelps and Pack [1959].

In oxygen, for $u<0.1 \mathrm{eV}$, the appropriate one term approximation is

$$
\nu_{m}(u)=7 \times 10^{-8} n\left(\mathrm{O}_{2}\right) u \mathrm{~cm}^{3} / \mathrm{sec}
$$


which is consistent with the observations to within \pm 10 percent [Phelps, 1960].

The electron collision frequency of air is obtained by adding the electron collision frequency due to each of the different components of the air. If only the major constituents, nitrogen and oxygen, are considered and $n\left(N_{2}\right)$ is replaced by $0.78 n$ and $n\left(\mathrm{O}_{2}\right)$ is replaced by $0.21 n$ where $n$ is the total molecular density in $\mathrm{cm}^{-3}$, then from (3) and (4) the one term approximation for the electron collision frequency in air is

$\left[\nu_{m}(u)\right]_{\mathrm{air}}=1.01 \times 10^{-7}$ un $\sec ^{-1}=8.40 \times 10^{7} p \mathrm{sec}^{-1}$

where $p$ is the atmospheric pressure expressed in $\mathrm{mm}$ Hg. The probable error associated with (5) is about \pm 10 percent; when the equation is applied to the ionospheric $D$ region, however, the probable error may be as large as +20 percent, -10 percent in the 30 to $40 \mathrm{~km}$ height range due to the presence of water-vapor.

\section{Cross Modulation Observations}

In the above discussion, the ionosphere collision frequency profiles obtained from rocket data have been mentioned. The method of cross modulation can also determine the electron collision frequency in the ionosphere. A direct measurement of $v$ can be obtained at a fixed height that is determined by the frequency of the wanted radio wave. To determine this height the reader is referred to the previous paper where the expression for the total cross modulation, observed on the received wanted wave, due to a pulse modulated disturbing wave is given as

$$
T=-\int_{0}^{h_{0}}\left[\frac{\partial K_{w}}{\partial \nu_{m}}\left(h^{\prime}\right)\right]\left[\Delta \nu_{m}\left(h_{0}, h^{\prime}\right)\right] d h^{\prime}
$$

where $h_{0}$ is the height of the leading edge of the upward traveling disturbing pulse and $K_{w}$ is the absorption coefficient for the wanted wave. The integrand changes sign at the height where $\nu_{m}=\frac{2 \pi f_{w} \pm \omega_{L}}{2.18}$, where $f_{w}$ is the frequency of the wanted wave and $\omega_{L}$ is the so-called "longitudinal component of the angular gyrofrequency of the electrons" [Benson, 1964]. This height is called the true crossover height. The observed crossover height, i.e., that value of $h_{0}$ that makes $T=0$, is greater than the true crossover height because it is determined by the height where the positive and negative contributions of the integrand cancel. The correction factors necessary to obtain the true crossover height from the observed crossover height can be approximated by computing the cross modulation expected for a model ionosphere. Under these conditions, the true crossover height is known from the initial conditions, the observed crossover height is given by the calculations, and the correction factor is given by the difference between the two. There are uncertainties that enter in this procedure, however, in that the exact electron density profile $N$ during the time of observation is not known and the term $\Delta v_{m}$ in the theory of ionospheric cross modulation is uncertain. At present, it appears that ionospheric cross modulation observations in the vicinity of the crossover region, i.e., the region where the observed cross modulation changes sign, agree with the original theory of cross modulation of Bailey and Martyn [1934]. The crossover point is then only slightly dependent on $N$ [Benson, 1964]. This theory was used to determine the correction factors for the three crossover points (corresponding to three values of $2 \pi f_{w} \pm \omega_{L}$ ) that have been observed at College, Alaska. The results are given below:

\begin{tabular}{l|c|c|c}
\hline \hline$f_{w}(\mathrm{Mc} / \mathrm{s}) *$ & Time & $\begin{array}{c}h_{0}(T=0) \\
(\mathrm{km})\end{array}$ & $\begin{array}{c}h^{\prime}\left(\frac{\partial K_{i v}}{\partial \nu_{m}}=\right. \\
0)(\mathrm{km}) * *\end{array}$ \\
\cline { 1 - 1 } $4.865(\mathrm{E})$ & $\begin{array}{c}1710 \\
\text { 22 August 1960 } \\
\text { 0240 }\end{array}$ & $70 \pm 4$ & $62 \pm 5$ \\
17.5 & $\begin{array}{c}\text { 29 June 1960 } \\
1230\end{array}$ & $57 \pm 3$ & $51 \pm 4$ \\
7 November 1961 & $48 \pm 3$ & $44 \pm 4$ \\
\hline
\end{tabular}

*(O) ordinary component, (E) extraordinary component; see Flock and Benson [1961, sec. 3.2] for the first two values and Benson [1962] for the last value.

**These values differ from the values given in the above references because a different method of approximating the correction factors was used earlier.

The corresponding values for $\nu_{m}$ are

$$
\begin{aligned}
& \nu_{m}=9.67 \times 10^{6} \mathrm{sec}^{-1} \text { at } 62 \pm 5 \mathrm{~km} \\
& \nu_{m}=1.84 \times 10^{7} \mathrm{sec}^{-1} \text { at } 51 \pm 4 \mathrm{~km} \\
& \nu_{m}=5.04 \times 10^{7} \mathrm{sec}^{-1} \text { at } 44 \pm 4 \mathrm{~km} .
\end{aligned}
$$

The above values are shown in figure 1, together with the ionospheric rocket results of Kane [1960] and a curve based on the laboratory measurements of Pack and Phelps [1961]. This curve follows from (5) when the values for the atmospheric pressure $p$ are obtained from the November 1956 daytime Fort Churchill rocket data of Lagow, Horowitz, and Ainsworth [1960, fig. 5]. The probable error associated with these pressure data ranges from \pm 5 percent at $30 \mathrm{~km}$ to \pm 8 percent at $80 \mathrm{~km}$. Combining this probable error with the probable error associated with (5) gives an overall probable error of approximately \pm 20 percent; this probable error is indicated in figure 1 by the shaded region above and below the curve representing the equation $\nu_{m}=8.40 \times 10^{7} p(\mathrm{~mm} \mathrm{Hg})$. The curve agrees (within the probable error) with the rocket data points from the November 1956 rocket flight of Kane. The collision frequency profile resulting: from the above equation is in agreement with the rocket data points from the July 1957 rocket flight of Kane if the July 1957 pressure profile of Lagow, Horowitz, and Ainsworth [1960, fig. 5] is used. (The July pressure values were considerably higher than the November pressure values, for example, at $65 \mathrm{~km}$ the difference was 45 percent.) 


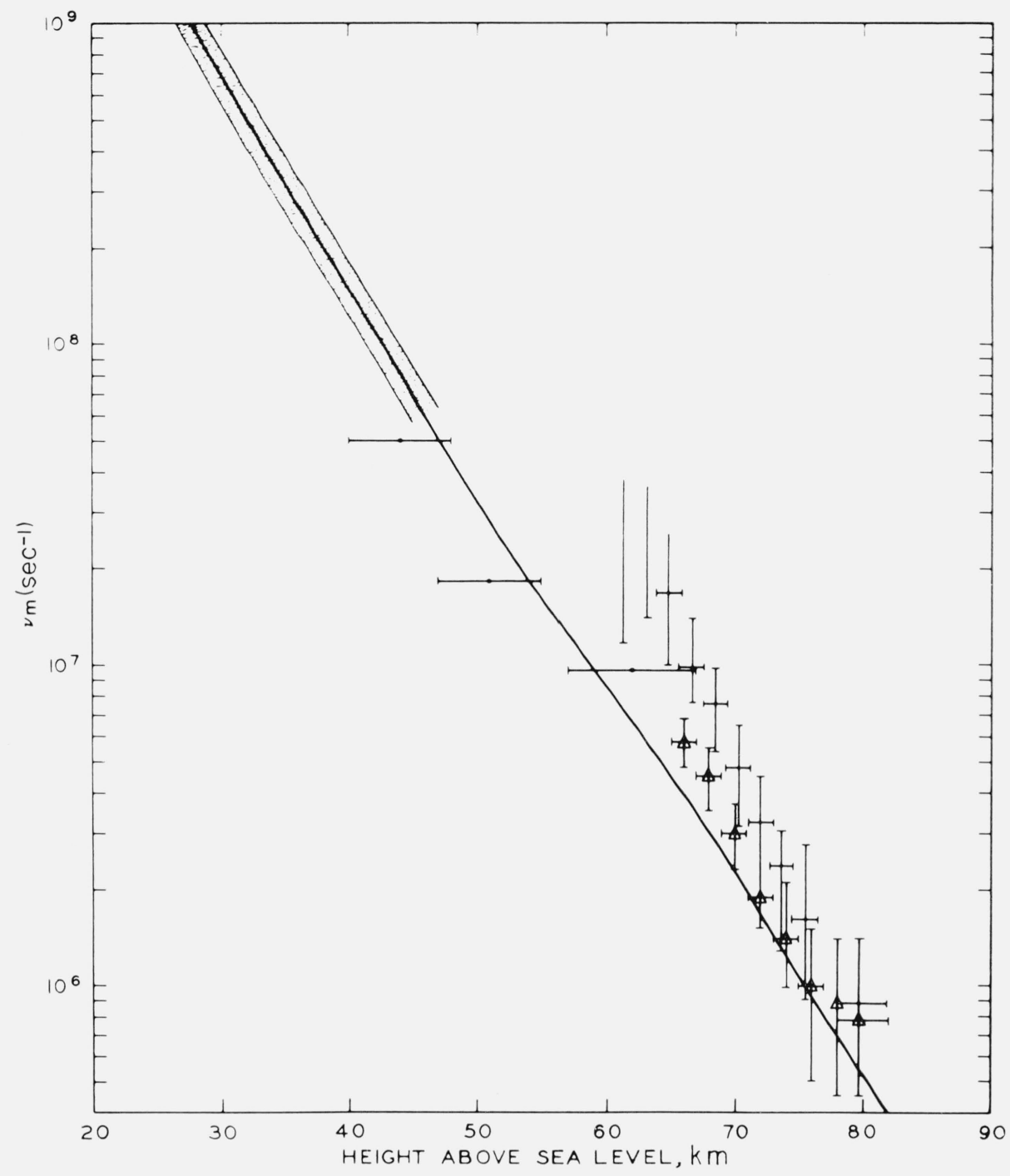

Figure 1. Electron collision frequency versus height.

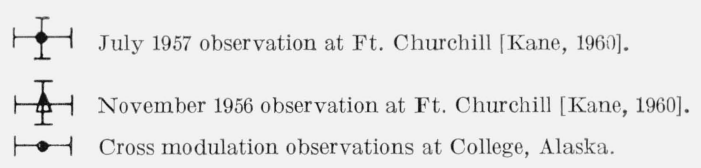

The solid curve, with probable error indicated, is derived from (5), i.e., $\nu_{m}=8.40 \times 10^{7} \mathrm{p}(\mathrm{mm} \mathrm{Hg})$, which is based on laboratory results in $N_{2}$ [Pack and Phelps, 1961], and the pressure values from a November $1956 \mathrm{Ft}$. Churchill rocket flight [Lagow et al., 1956, fig. 5].

The three horizontal lines in figure 1 represent the values of the electron collision frequency as determined from the cross-modulation experiment at College, Alaska. The daytime November 1961 value of $\nu_{m}$ at $44 \pm 4 \mathrm{~km}$ agrees with the curve which is based on (5) and the daytime November 1956 atmospheric pressure above Fort Churchill, Canada.

The daytime August 1960 value of $\nu_{m}$ at $62 \pm 5 \mathrm{~km}$ agrees with the daytime July 1957 rocket measurements of Kane [1960] above Fort Churchill, Canada.
This value also agrees very well with (5) when the July 1957 Fort Churchill pressure values of Lagow et al., (1960, fig. 5) are used.

The 0240,29 June 1960 value of $\nu_{m}$ at $51 \pm 4 \mathrm{~km}$ cannot be compared directly with the daytime November or the daytime July Fort Churchill measurements. Rather, it must be considered as the appropriate $\nu_{m}$ corresponding to the atmospheric pressure above College, Alaska, at the time of observation. 
In summary, (5), which is based on the laboratory studies in $\mathrm{N}_{2}$ by Pack and Phelps [1961], is in agreement with the Fort Churchill, Canada, rocket measurements of Kane [1960] and the College, Alaska, cross-modulation observations, when the appropriate values for the atmospheric pressure are used.

I am grateful to Dr. A. V. Phelps for his helpful correspondence.

This work was supported by the Air Force Cambridge Research Laboratories Office of Aerospace Research under Contract No. AF 19(604)-8833 and, in part, by the Defense Atomic Support Agency, Washington, D.C., under Web No. 04.0063.

\section{References}

Bailey, V. A., and D. F. Martyn (1934), The influence of electric waves on the ionosphere, Phil. Mag. 18, 369-386.

Benson, R. F. (1962), Cross modulation of cosmic noise, J. Geophys. Res. 67, 2569-2572.

Benson, R. F. (Oct. 1964), A discussion of the theory of ionospheric cross modulation, Radio Sci. J. Res. NBS/URSI 68D, No. 10, 1109-1122.
Flock, W. L., and R. F. Benson (Aug. 1961), Experiment Luxembourg final rept., Contract AF 19(604)-3880; Univ. of Alaska, Geophys. Institute Rept. 121.

Kane, J. A. (1961), Reevaluation of ionosphere electron densities and collision frequencies derived from rocket measurements of refractive index and attenuation. NASA Technical Note D-503, Nov. 1960. J. Atmospheric Terrest. Phys. 23, 338-347.

Lagow, H. E., R. Horowitz, and J. Ainsworth (1960), Results of IGY atmospheric density measurements above Fort Churchill, Space Research, Proceedings of the First International Space Science Symposium, Nice, Jan. 11-16, 1960, ed. by H. Kallmann, 164-174.

Pack, J. L., and A. V. Phelps (1961), Drift velocities of slow electrons in helium, neon, argon, hydrogen, and nitrogen, Phys. Rev. 121, 798-806.

Phelps, A. V. (1960), Propagation constants for electromagnetic waves in weakly ionized, dry air, J. Appl. Phys. 31, 1723-1729.

Phelps, A. V., and J. L. Pack (1959), Electron collision frequencies in nitrogen and in the lower ionosphere, Phys. Rev. Letters 3, 340-342.

Stroud, W. G., W. Nordberg, W. R. Bandeen, F. L. Bartman, and P. Titus (1960), Rocket-grenade measurements of temperatures and winds in the mesosphere over Churchill, Canada, J. Geophys. Res. 65, 2307-2323.

(Paper 68D10-411) 